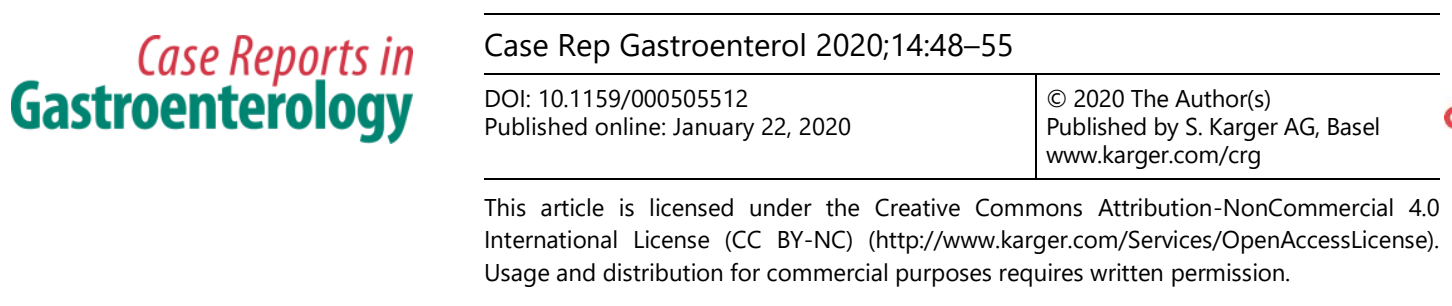

\title{
Peroral Endoscopic Myotomy in a Patient with Achalasia Cardia with Prior Heller's Myotomy
}

\author{
Muhammad Miftahussurur $^{\mathrm{a}} \quad$ Manu Tandan $^{\mathrm{b}}$ Dadang Makmun $^{\mathrm{c}}$ \\ Zaheer $\mathrm{Nabi}^{\mathrm{b}}$ \\ aDivision of Gastroentero-Hepatology, Department of Internal Medicine, Faculty of \\ Medicine, Dr. Soetomo Teaching Hospital, Institute of Tropical Disease, Universitas \\ Airlangga, Surabaya, Indonesia; ${ }^{b}$ Asian Institute of Gastroenterology, Hyderabad, India; \\ 'Division of Gastroenterology, Department of Internal Medicine, Faculty of Medicine, \\ Universitas Indonesia, Cipto Mangunkusumo National General Hospital, Jakarta, Indonesia
}

\section{Keywords}

Achalasia cardia · Peroral endoscopic myotomy · Post Heller's myotomy

\begin{abstract}
Achalasia cardia is an esophageal myenteric plexus disorder characterized by absence of or incomplete lower esophageal sphincter relaxation and esophageal aperistalsis; Heller's myotomy is the main treatment of choice due to a lower failure rate. Recently, peroral endoscopic myotomy (POEM) has been reported as an alternative treatment for achalasia due to persistent symptoms after Heller's myotomy. An Indian male, aged 18 years, was admitted to the hospital due to dysphagia which had started more than 3 years ago. He also complained of occasional regurgitation and retrosternal pain with Eckardt score 6 . Heller's myotomy was performed 2 years ago. Barium swallow showed achalasia cardia and upper gastrointestinal endoscopy found liquid residue and resistance at the gastroesophageal junction. Esophageal manometry is concluded as achalasia cardia type II with a median integrated relaxation pressure (IRP) of $25.6 \mathrm{~mm} \mathrm{Hg}$. He underwent POEM; with the help of a submucosal tunnel, an extension of up to $1 \mathrm{~cm}$ beyond the gastroesophageal junction could be achieved with a posterior orientation of myotomy. There were no adverse events after the POEM procedure. He was treated with a soft diet for 10 days and other supportive treatments. Following POEM, barium swallow
\end{abstract}


showed a significant improvement and esophageal manometry exhibited that the basal lower esophageal sphincter pressure was normal with complete relaxation on swallowing and normal median IRP. The post-procedure Eckardt score was 0 . We reported an achalasia patient who received POEM after unsuccessful Heller's myotomy and showed clinical improvement.

(C) 2020 The Author(s)

Published by S. Karger AG, Basel

\section{Introduction}

Achalasia cardia is a neurodegenerative disorder of the esophageal myenteric plexus characterized by absence of or incomplete lower esophageal sphincter relaxation and esophageal aperistalsis, resulting in symptoms such as dysphagia, regurgitation, and chest pain.

In achalasia, the diagnostic procedure is performing barium esophagogram that is showing a pathognomonic "bird's beak" sign in the distal esophagus while upper gastrointestinal endoscopy is showing a narrow gastroesophageal junction. Esophageal manometry is the gold standard in diagnosing achalasia and high-resolution manometry with esophageal pressure topography that could increase the specificity and sensitivity [1]. High-resolution manometry can divide achalasia into three subtypes [2]. Type I is characterized by the loss of smooth muscle contractility in the esophageal body and the lack of compartmentalized esophageal pressurization, whilst in type II achalasia, circular muscle excitation is sufficient and the longitudinal muscle contraction is preserved, resulting in periods of compartmentalized esophageal pressurization or compression. Thus, it shows good treatment response by lower esophageal sphincter pressure reduction. The last and least frequent achalasia type (type III) shows a spastic contraction of the distal esophagus that appears in $20 \%$ of swallows [2].

Treatment of achalasia include medical management, pneumatic balloon dilatation, botulinum toxin injection, esophagectomy, and laparoscopic Heller's myotomy. Pneumatic balloon dilatation is useful for type I and type II patients, whereas Heller's myotomy with partial fundoplication is effective for type II and III achalasia [3]. Although balloon dilatation has the same efficacy for the primary outcome, the lower failure rate of Heller's myotomy makes it the treatment of choice with a low surgical risk for the patient. Recently, peroral endoscopic myotomy (POEM) has become the preferred alternative treatment due to its minimally invasive technique [16]. The short-term efficacy rates of POEM are similar to Heller's myotomy and the response rate was over $90 \%$ [4]. Furthermore, it also presented excellent symptom control over a 3-year period in all 3 types of achalasia, as previously reported in the cohort study [5, 6]. POEM was not only associated with a shorter duration of operation, hospital stay, and recovery time, but also with less blood loss, postoperative pain, and analgesic use. POEM was also found to be safe and effective in patients with persistent symptoms after Heller's myotomy, although the rate of clinical success was lower in patients without prior Heller's myotomy (81 vs. 94\%, respectively) [7]. We reported the case of an achalasia patient who received POEM after unsuccessful Heller's myotomy.

\section{Case Report}

An 18-year-old Indian male was admitted to the hospital due to dysphagia which had started more than 3 years ago. He also complained of occasional regurgitation and retrosternal pain. No weight loss was detected. Two years ago, laparoscopic Heller's myotomy was carried out. On physical examination, the patient was not pale, no icterus and pedal edema were 
found. The thorax was normal, the abdomen was soft, non-tender, and there were no ascites. The pre-procedure Eckardt score was 6.

His routine blood investigations showed a normal hemogram. Liver and renal function test, serum electrolytes, blood glucose, and coagulation test were all normal. Thyroid-stimulating hormone, C-reactive protein, and erythrocyte sedimentation rate were normal. Hepatitis and acquired immunodeficiency syndrome (AIDS) viral markers were negative.

The X-ray showed bilateral lung parenchyma and cardiac examination appeared normal. Barium esophagography was performed 1, 2, and 5 min after ingestion solution showed a "bird's beak" appearance with a dilated esophageal body (Fig. 1). Upper gastrointestinal endoscopy exhibited esophagus with liquid residue and resistance at the gastroesophageal junction suggestive of achalasia cardia. Esophageal manometry showed that the basal lower esophageal sphincter pressure was normal with incomplete relaxation on swallowing. On 5$\mathrm{mL}$ wet swallows, normal esophageal peristaltic wave was absent with panesophageal pressurization in $>20 \%$ of swallows. Median integrated relaxation pressure (IRP) was more than $15 \mathrm{~mm}$ Hg. Esophageal manometry was concluded as achalasia cardia type II following Heller's myotomy with a median IRP of $25.6 \mathrm{~mm} \mathrm{Hg}$ (Fig. 2). He was diagnosed with type II achalasia.

After cardiologist and pulmonologist clearance, he underwent POEM. The patient was intubated in supine position. The gastroesophageal junction was at $45 \mathrm{~cm}$. A vertical mucosal incision was made posteriorly at the 5 o'clock position (Fig. 3). After submucosal injection of saline at $35 \mathrm{~cm}$ using a needle knife and IT knife, submucosal dissection was performed using a triangle-tip knife (Olympus, Tokyo, Japan) with a spray coagulation mode. Along with the help of a submucosal tunnel, an extension of up to $1 \mathrm{~cm}$ beyond the gastroesophageal junction could be achieved. Myotomy was performed with posterior orientation using a triangle-tip knife (spray coagulation mode, ERBE electrosurgical generator ICC 300, ERBE) extending from $37 \mathrm{~cm}$ up to $2 \mathrm{~cm}$ beyond the gastroesophageal junction. Mucosal incision was closed using hemoclips.

He was treated with a soft diet for 10 days followed by a normal diet, proton pump inhibitor, prokinetic, and other supportive treatments. Following POEM, barium swallow showed significant improvement. The changes of barium esophagogram pre- and post-POEM can be seen in Figure 1. He was able to tolerate oral liquids by day 2 and a soft diet by day 3 after the procedure. He was being discharged in a stable condition. The nature of underlying diseases, long-term prognosis, and complications have been explained in detail to the patient and his attendants.

After 1 month, re-esophageal manometry was performed. It showed that the basal lower esophageal sphincter pressure was normal with complete relaxation on swallowing. On 5-mL wet swallows, normal esophageal peristaltic wave was absent. The median IRP was $10.3 \mathrm{~mm}$ $\mathrm{Hg}$ (normal) and the post-procedure Eckardt score was 0.

\section{Discussion}

We reported an achalasia patient who had undergone unsuccessful Heller's myotomy. After the POEM procedure, his symptoms were reduced and he had a normal IRP and Eckardt score. There are several reasons for the recurrence of achalasia symptoms after Heller's myotomy including inadequate myotomy (53\%), fundoplication failure (26\%), extensive fibrosis (19\%), and mucosal stricture (2\%) [8]. Appearance of fibrosis in the healing or incomplete division of muscle fiber in the distal site of myotomy creating a residual high-pressure zone 
that might be the reasons for inadequate myotomy. Thus, it is suggested that Heller's myotomy size should not be less than $4 \mathrm{~cm}$ on the esophagus. On the stomach, the myotomy size should be more than $1 \mathrm{~cm}$ but less than $2 \mathrm{~cm}$ to deal with reflux. The POEM procedure consists of making a submucosal tunnel of $10 \mathrm{~cm}$ proximal to the gastroesophageal junction of the esophagus and myotomy of circular muscle layers approximately $2 \mathrm{~cm}$ into the cardia [9]. The longer myotomy size is often necessary in diffuse esophageal spasm jackhammer esophagus and type III achalasia [10].

Pneumatic balloon dilatation is one of the options in case of a treatment failure in patients who had undergone Heller's myotomy due to its safe method and excellent short-term response rates; however, there was a high number of evidence of long-term relapse requiring re-dilatation [11]. A repetition of Heller's myotomy is also an option; however, it resulted in a longer length of hospital stay, less magnitude of improvement in symptoms (dysphagia, choking, and coughing), and higher conversion rate to an "open" myotomy than for patients undergoing their first myotomy [12]. POEM is a suitable procedure for naïve patients, patients with a prior treatment failure as well as for patients with sigmoid-type achalasia (although a skilled endoscopist is required) due to its safety and efficacy. Patients with end-stage achalasia (tortuous or sigmoid esophagus type II, classified based on computed tomography) may require esophagectomy [13].

POEM is more advantageous than Heller's myotomy because the myotomy orientation depends on the operator's preferences and clinical situation. The orientation can be a posterior (the 5 o'clock direction) or anterior (1-2 o'clock position) approach, but should not be lateral one. The lateral side of the esophagus is weak; thus, the lateral approach of POEM might cause diverticula [10]. It was reported that the anterior approach of POEM has equal efficacy as the posterior approach, but the anterior approach has a higher rate of mucosal injury (20 vs. 3.3\%). On the other hand, the posterior approach has a higher acid exposure than the anterior one (2.98 vs. $13.99 \%$ ) [9]. POEM after failed surgical myotomy also has a short-term clinical success rate of $92-100 \%$ by preventing previous myotomy scars including submucosal fibrosis [10]. Thus, anterior orientation scars can be avoided by performing posterior myotomy [14].

We can predict the treatment outcome and recurrence rate of achalasia by gastrointestinal endoscopy and medical interview (also barium swallow and high-resolution monometer, if necessary) at 2-3 months after procedures, then annually [10]. The questions asked in the medical interview include changes in the amount of meals consumed and changes in body weight after the procedure, compared to before the procedure. Esophageal symptoms such as dysphagia and chest pain were also included in the interview and can be measured by Eckardt scores [13]. The opening of the esophagogastric junction by gastrointestinal endoscopy should also be evaluated and compared with pre-POEM.

Several adverse events of POEM were reported, such as mucosal perforation, pneumothorax, pleural effusion, insufflation-related problem, and bleeding (massive intraprocedural hemorrhage and delayed submucosal hematoma) $[9,10]$. Injury on the branch of the left gastric artery on the gastric side can cause bleeding in the proximal or distal cardia during the tunneling or myotomy [9]. Attention should be paid to perforation due to the connection between submucosal tunnel gas, the mediastinum, and peritoneum. This perforation can be detected intraprocedurally and closed with clips. The POEM procedure, especially posterior myotomy, involves cutting the clasps and sling fiber at the gastroesophageal junction resulting in an increase in reflux that should be minimized [9]. Even though reflux esophagitis and symptomatic gastroesophageal reflux disease were well controlled by proton pump inhibitors for 3 months [15], the double-scope method can be used to reduce the reflux. Using the double- 
scope method, incomplete and prolonged myotomy could be avoided toward the gastric side by direct visualization of the tip of the submucosal endoscope through the pediatric scope in the stomach. In conclusion, we reported an achalasia patient who received POEM after unsuccessful Heller's myotomy. Following the procedure, he showed clinical improvement with normal manometry and Eckardt score.

\section{Statement of Ethics}

Informed consent was obtained for this case report. Ethics approval was not needed for this paper.

\section{Disclosure Statement}

The authors have no conflicts of interest to declare.

\section{Funding Sources}

There are no funding sources to report.

\section{Author Contributions}

Muhammad Miftahussurur and Zaheer Nabi performed data acquisition and drafted the manuscript. Muhammad Miftahussurur, Manu Tandan, and Dadang Makmun supervised and provided critical revision of the manuscript. All authors read and approved the final manuscript.

\section{References}

1 Zaninotto G, Leusink A, Markar SR. Management of achalasia in 2019. Curr Opin Gastroenterol. 2019 Apr;35(4):356-62.

2 Kahrilas PJ, Bredenoord AJ, Fox M, Gyawali CP, Roman S, Smout AJ, et al.; International High Resolution Manometry Working Group. The Chicago Classification of esophageal motility disorders, v3.0. Neurogastroenterol Motil. 2015 Feb;27(2):160-74.

3 O'Neill OM, Johnston BT, Coleman HG. Achalasia: a review of clinical diagnosis, epidemiology, treatment and outcomes. World J Gastroenterol. 2013 Sep;19(35):5806-12.

4 Von Renteln D, Fuchs KH, Fockens P, Bauerfeind P, Vassiliou MC, Werner YB, et al. Peroral endo scopic myotomy for the treatment of achalasia: an international prospective multicenter study. Gastroenterology. 2013;145(2):309-11.e1-3.

5 Inoue H, Sato H, Ikeda H, Onimaru M, Sato C, Minami H, et al. Per-Oral Endoscopic Myotomy: A Series of 500 Patients. J Am Coll Surg. 2015 Aug;221(2):256-64.

6 Teitelbaum EN, Soper NJ, Santos BF, Arafat FO, Pandolfino JE, Kahrilas PJ, et al. Symptomatic and physiologic outcomes one year after peroral esophageal myotomy (POEM) for treatment of achalasia. Surg Endosc. 2014 Dec;28(12):3359-65.

7 Ngamruengphong S, Inoue H, Ujiki MB, Patel LY, Bapaye A, Desai PN, et al. Efficacy and Safety of Peroral Endoscopic Myotomy for Treatment of Achalasia After Failed Heller Myotomy. Clin Gastroenterol Hepatol. 2017 Oct;15(10):1531-7.e3. 
8 Veenstra BR, Goldberg RF, Bowers SP, Thomas M, Hinder RA, Smith CD. Revisional surgery after failed esophagogastric myotomy for achalasia: successful esophageal preservation. Surg Endosc. 2016 May;30(5):1754-61.

9 Ramchandani M, Nabi Z, Reddy DN, Talele R, Darisetty S, Kotla R, et al. Outcomes of anterior myotomy versus posterior myotomy during POEM: a randomized pilot study. Endosc Int Open. 2018 Feb;6(2):E190-8.

10 Inoue H, Shiwaku H, Iwakiri K, Onimaru M, Kobayashi Y, Minami H, et al. Clinical practice guidelines for peroral endoscopic myotomy. Dig Endosc. 2018 Sep;30(5):563-79.

11 Kumbhari V, Behary J, Szczesniak M, Zhang T, Cook IJ. Efficacy and safety of pneumatic dilatation for achalasia in the treatment of post-myotomy symptom relapse. Am J Gastroenterol. 2013 Jul;108(7):1076-81.

12 Wood TW, Ross SB, Ryan CE, Bowman TA, Jacobi BL, Konstantinidis MG, et al. Reoperative Heller myotomy: more pain, less gain. Am Surg. 2015 Jun;81(6):637-45.

13 Ramchandani M, Nageshwar Reddy D, Nabi Z, Chavan R, Bapaye A, Bhatia S, et al. Management of achalasia cardia: expert consensus statements. J Gastroenterol Hepatol. 2018 Aug;33(8):1436-44.

14 Bukhari M, Chavez YH, Chen YI, Brewer Gutierrez OI, Khashab MA. A third myotomy with peroral endoscopic myotomy after two failed Heller myotomies. Endoscopy. 2017 Nov;49(11):1110-2.

15 Shiwaku H, Inoue H, Sasaki T, Yamashita K, Ohmiya T, Takeno S, et al. A prospective analysis of GERD after POEM on anterior myotomy. Surg Endosc. 2016 Jun;30(6):2496-504.

16 Inoue $\mathrm{H}$, Minami H, Kobayashi Y, et al. Peroral endoscopic myotomy (POEM) for esophageal achalasia. Endoscopy. 2010 Apr;42(4):265-71. 


\section{Case Reports in Gastroenterology}

\begin{tabular}{l|l}
\hline Case Rep Gastroenterol 2020;14:48-55 \\
\hline DOI: 10.1159/000505512 & $\begin{array}{l}\text { ○ 2020 The Author(s). Published by S. Karger AG, Basel } \\
\text { www.karger.com/crg }\end{array}$ \\
\hline
\end{tabular}

Miftahussurur et al.: POEM on Prior Myotomy Achalasia Patient
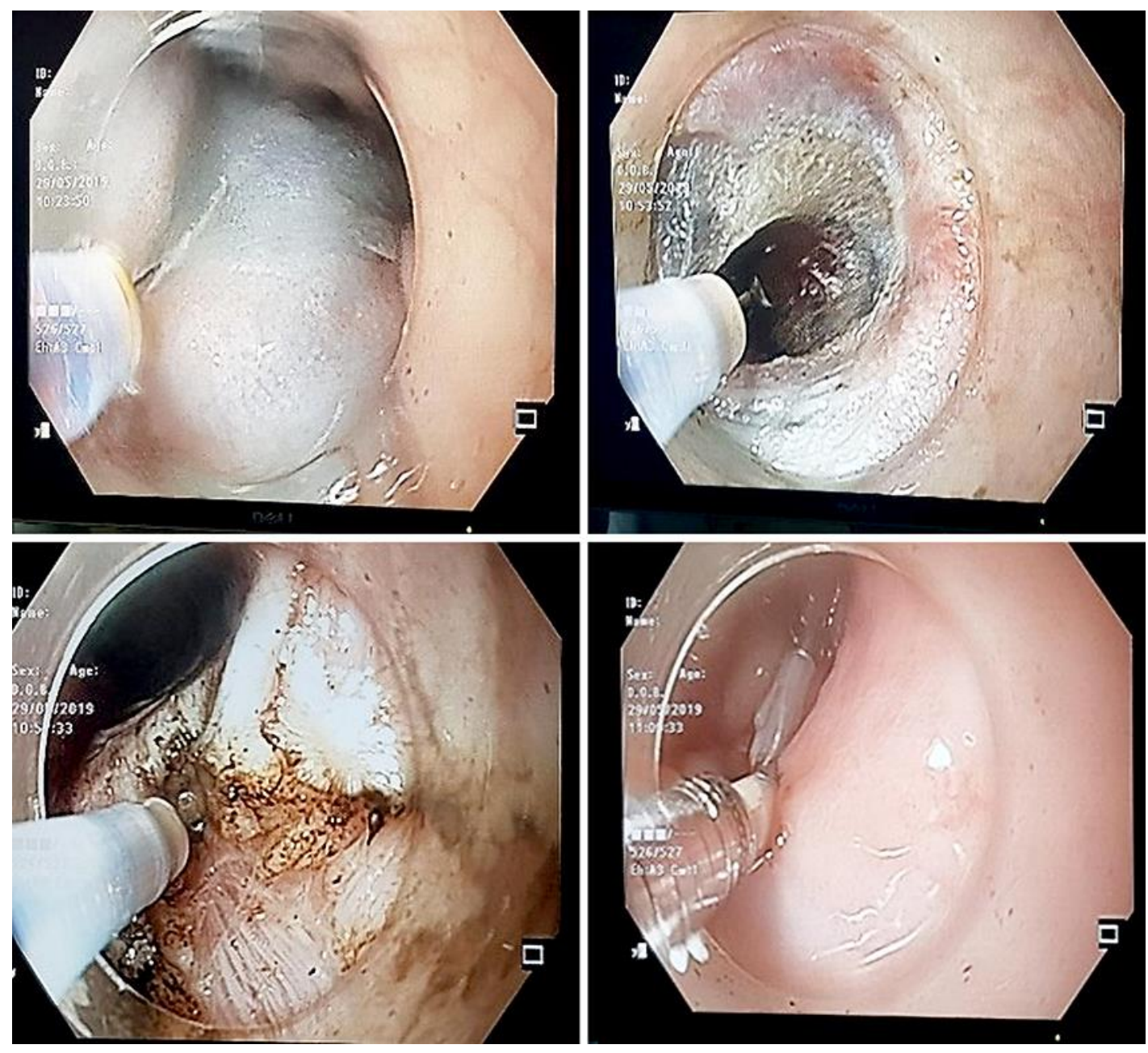

Fig. 1. Barium esophagogram pre- and post-POEM.

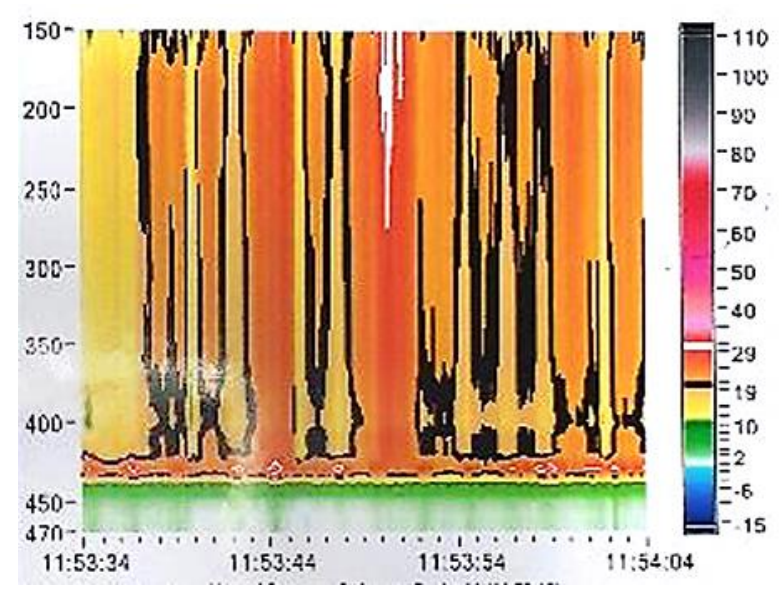

Fig. 2. High-resolution manometry pre-POEM found achalasia type II with a median integrated relaxation pressure of $25.6 \mathrm{~mm} \mathrm{Hg}$. 


\begin{tabular}{|c|c|c|}
\hline \multirow{2}{*}{$\begin{array}{l}\text { Case Reports in } \\
\text { Gastroenterology }\end{array}$} & \multicolumn{2}{|c|}{ Case Rep Gastroenterol 2020;14:48-55 } \\
\hline & DOI: 10.1159/000505512 & $\begin{array}{l}\text { O } 2020 \text { The Author(s). Published by S. Karger AG, Basel } \\
\text { www.karger.com/crg }\end{array}$ \\
\hline
\end{tabular}
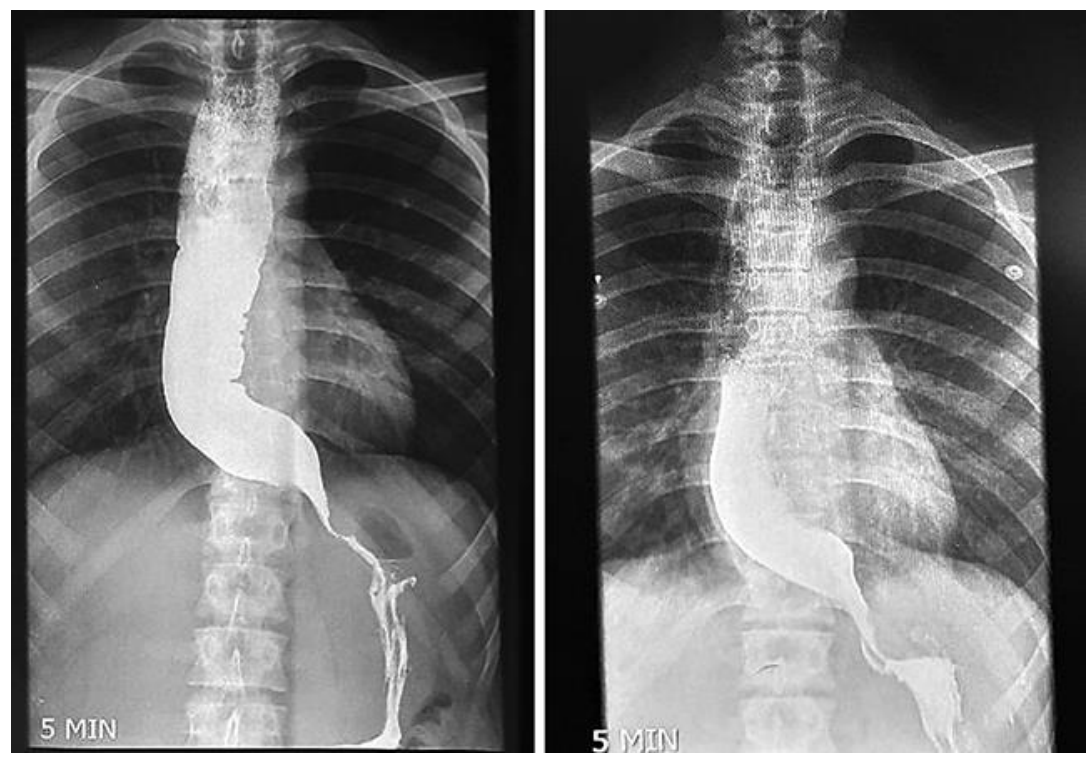

Fig. 3. POEM with a posterior approach (mucosal incision at the 5 o'clock position). 